\title{
Análise não-paramétrica de dados ordinais com medidas repetidas
}

\section{Patrícia Rosa}

\author{
Dissertação apresentada ao \\ INSTITUTO DE MATEMÁTICA E ESTATÍSTICA \\ da \\ UNIVERSIDADE DE SÃO PAULO \\ para a obtenção do grau de mestre em Estatística
}

Área de Concentração: Estatística

Orientador: Prof. Dr. Julio da Motta Singer

São Paulo, novembro de 2001 


\title{
Análise não-paramétrica de dados ordinais com medidas repetidas
}

\begin{abstract}
Este exemplar corresponde à redação
final da dissertação devidamente corrigida e defendida por Patrícia Rosa e aprovada pela comissão julgadora.
\end{abstract}

São Paulo, novembro de 2001

Banca Examinadora:

- Prof. Dr. Julio da Motta Singer (Orientador) - USP

- Prof. Dr. Dalton Francisco Andrade - UFC

- Prof. Gulherme Rosa - UNESP 
A minha tia Célia

Regina e a minha avó Maria Aparecida 


\section{Agradecimentos}

Gostaria de agradecer às pessoas que contribuíram para a realização deste trabalho, em especial:

- Ao Professor Julio da Motta Singer, pelo apoio, disposição e paciência que dedicou a este trabalho e pela contribuição em minha formação desde o curso de graduação;

- A Denise e Daniela pela amizade e pelo incentivo constante;

- À Priscila pela colaboração e incentivo;

- A Laércio Vicente e Carlos Dadoorian pelo tempo que me permitiram dedicar a este trabalho. 


\section{Resumo}

Neste trabalho comparamos duas metodologias de análise de dados ordinais coletados em experimentos com medidas repetidas. A primeira considera a atribuição de escores às categorias de resposta e a utilização de modelos paramétricos para comparar as médias destes escores. Esta técnica é amplamente utilizada uma vez que a metodologia de Análise de Variância para Medidas Repetidas na qual se baseia é bastante difundida e vários pacotes estatísticos disponibilizam rotinas de ajuste e análise. No entanto, sua utilização é criticada devido às suposições bastante restritivas dessa classe de modelos, como a continuidade da variável resposta, distribuição normal dos dados e estrutruras especiais para as matrizes de covariância. Por essas razões, uma metodologia alternativa, baseada em técnicas não-paramétricas que apresenta suposições menos restritivas é descrita. Esta metodologia utiliza os postos das observações para estudar os efeitos dos fatores na distribuição da variável resposta. Os procedimentos utilizados para investigar as hipóteses de interesse são baseados na distribuição assintótica das estatísticas propostas e a exatidão das aproximações pode ser insatisfatória no caso de experimentos com amostras pequenas. Por intermédio de estudos de simulação, comparamos as taxas de erro Tipo-I e o poder dos testes para as técnicas paramétrica e não-paramétrica, sob planejamentos balanceados. Conclusões similares são obtidas utilizando modelos paramétricos para escores equiespaçados e a estatística tipo-ANOVA para o modelo não-paramétrico. Indicam ainda diferenças nas conclusões da análise paramétrica quando vários sistemas de pontuação para a mesma variável ordinal são utilizados. 


\begin{abstract}
We consider two methodologies to compare treatments in designs with repeated measures of ordered categorical data. The first one compares means of numerical scores assigned to the outcome levels of the response variable using classical parametric ANOVA models. Although it is widely known and available in many statistical packages, this approach is generally criticized since it requires an underlying Gaussian distribution for the response variable as well as special covariance matrix structures. An alternative technique based on non-parametric models with less restrictive hypotheses is considered. The rank of each observation is used to study the effects of the treatment factors on the response distribution. The analysis procedure is based on the asymptotic distributions of the test statistics proposed. By means of simulation studies we compare the two approaches with respect to the type-I error rate and the power of the test statistics for different sample sizes. Similar conclusions are obtained under the parametric model with equally spaced scores and under the non-parametric ANOVA-type statistic. The results obtained under the parametric model depend on scores assigned to the ordinal response variable.
\end{abstract}




\section{Índice}

1. Introdução

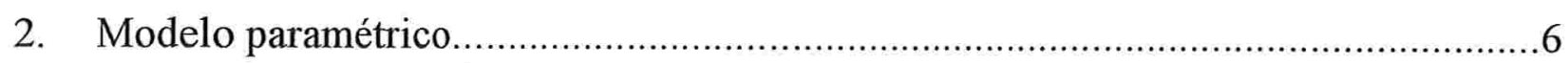

3. Definição do modelo não-paramétrico e das hipóteses de interesse..........................10

4. Estimação dos efeitos de tratamentos e estatística de teste........................................14

4.1. Distribuição dos estimadores dos efeitos de tratamento.......................................16

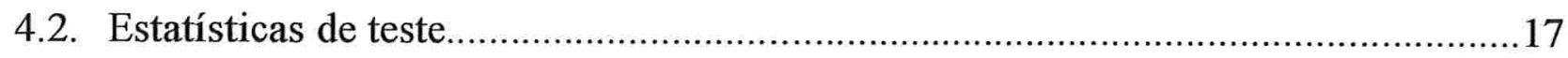

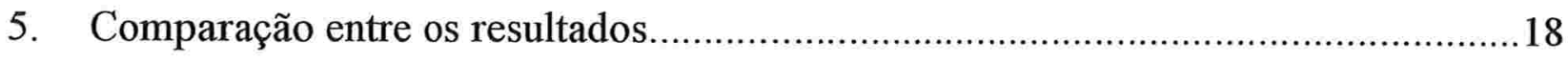

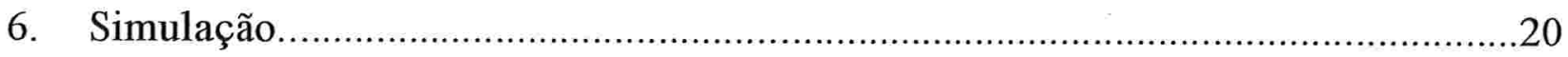

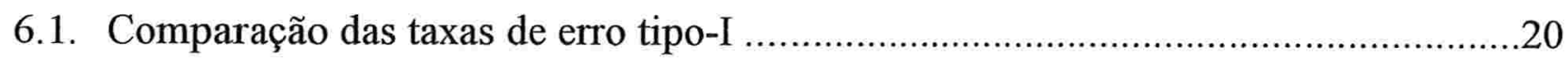

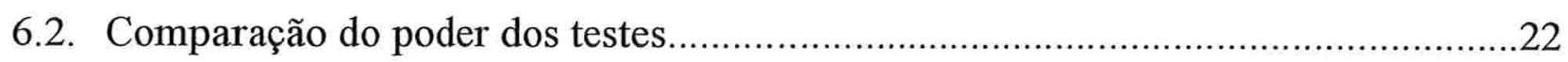

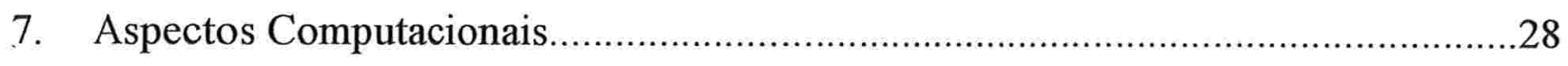

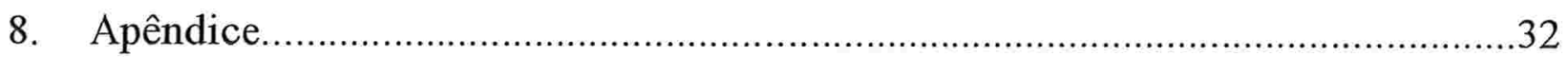

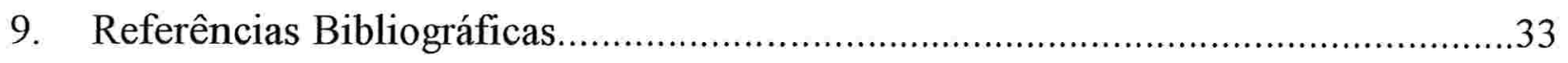




\section{Introdução}

Em estudos com medidas repetidas, as observações são coletadas em uma mesma unidade amostral, sob diferentes condições e sua principal vantagem é que eles dão margem à uma maior precisão na comparação dos diferentes tratamentos, pois a variabilidade entre as unidades amostrais é convenientemente controlada. Além disso, é uma alternativa vantajosa nos casos em que o número de unidades amostrais é reduzido. Em geral, o objetivo de estudos com essa estrutura é avaliar o comportamento de uma ou mais variáveis respostas sob os diferentes tratamentos envolvidos.

Um exemplo de estudo conduzido sob esse enfoque foi realizado na Faculdade de Odontologia da Universidade de São Paulo (Witzel et al., 2000) e consiste de um experimento realizado in vitro para avaliar a microinfiltração em superfícies dentárias tratadas com diferentes tipos de adesivos. Os tratamentos foram definidos pela combinação dos níveis dos fatores Material (Allbond, Optibond e Scotchbond), Campo (seco e úmido) e Ciclagem (controle, térmica e termo-mecânica) e aplicados em molares e pré-molares íntegros, extraídos por razões de cunho odontológico.

Foram utilizados 72 dentes, 4 em cada tratamento. Após a aplicação do adesivo e dos procedimentos de ciclagem, cada dente foi seccionado em 6 superfícies, que foram classificadas quanto à profundidade de possíveis microinfiltrações numa escala ordenada de 5 níveis variando de "sem microinfiltração" a "com microinfiltração de maior intensidade". Cada seção foi avaliada, independentemente, por 3 examinadores devidamente treinados.

O planejamento experimental envolve 4 fatores cruzados (Material, Campo, Ciclagem e Examinador) com medidas repetidas em um deles (Examinador) e o objetivo do experimento é estudar a influência dos fatores Material, Campo e Ciclagem na profundidade de microinfiltração, considerando as possíveis discrepâncias entre as classificações atribuídas pelos diferentes avaliadores.

As técnicas geralmente utilizadas para análise de dados com medidas repetidas são baseadas em Modelos Lineares, como de Análise de Variância ou Análise de Regressão. Na maior parte dos casos, supõe-se que a variável resposta tem distribuição Gaussiana. Efeitos aleatórios podem ser incorporados no modelo para representar correlações entre observações tomadas numa mesma unidade de investigação; a influência dos tratamentos 
na distribuição da variável resposta é medida pelos efeitos principais e interações, definidos como combinações lineares das médias das distribuições assumidas para os dados. A análise de dados com essas características tem sido foco de amplo esforço de pesquisa e o leitor poderá consultar Crowder and Hand (1990), Diggle et al. (1994) ou Singer and Andrade (2000) para ter uma visão pormenorizada sobre o assunto.

No caso em que a escala de avaliação é ordinal, uma alternativa é atribuir escores às classes de resposta, transformando-as de forma a possibilitar a aplicação das técnicas usuais. No exemplo citado anteriormente, a escala ordenada dos níveis de microinfiltração foi transformada em uma escala quantitativa através da atribuição dos escores de microinfiltração 0, 1, 2, 3 ou 4. Esta metodologia de análise, embora amplamente utilizada, é bastante criticada, principalmente porque métodos apropriados para análise de variáveis contínuas são utilizados numa situação em que a variável é discreta. A escala impõe uma relação de distância entre as categorias de resposta que é arbitrariamente definida, como no exemplo. Por essas razões, procedimentos baseados em suposições menos restritivas constituem uma boa fonte de pesquisas de análises alternativas.

Neste trabalho apresentamos uma metodologia baseada numa modelagem não-paramétrica para análise de dados ordinais com medidas repetidas, e comparamos os resultados com aqueles obtidos a partir dos modelos paramétricos mencionados anteriormente. $\mathrm{O}$ método é baseado em Akritas and Arnold (1994), que definem efeitos de tratamentos em termos de probabilidades associadas às distribuições marginais da variável resposta e propõem estatísticas baseadas em postos para testar as hipóteses de interesse. A extensão para planejamentos com observações correlacionadas e variáveis resposta ordinais, apresentada neste trabalho, é baseada na proposta de Akritas and Brunner (1997).

Para efeito de comparação, o modelo e os resultados da análise usual dos dados do estudo de microinfiltração dentária são reproduzidos na Seção 2. O modelo não-paramétrico é apresentado na Seção 3, juntamente com a definição dos efeitos de tratamento e a construção das hipóteses de interesse. Na Seção 4, discutimos a estimação desses efeitos, a distribuição dos estimadores e apresentamos estatísticas para testar as hipóteses especificadas na Seção 3. A aplicação da técnica aos dados do exemplo e a comparação dos resultados dos modelos paramétrico e não-paramétrico são apresentados na Seção 5 . A Seção 6 apresenta os resultados de uma simulação delineada para estudar o comportamento das 
estatísticas de teste associadas às duas técnicas ilustradas na Seção 5. Finalmente, na Seção 7, tratamos dos aspectos computacionais para o ajuste do modelo não-paramétrico.

\section{Modelo Paramétrico}

Os dados do estudo citado na Seção 1 foram originalmente analisados através de modelos de Análise de Variância para o Escore de microinfiltração, utilizando todos os fatores descritos anteriormente. Para efeito de comparação com o modelo proposto nesse trabalho, reproduziremos nesta seção a análise paramétrica realizada para os escores atribuídos à uma das superfícies (central), utilizando somente 3 fatores (Material, Campo e Examinador). As observações coletadas são apresentadas na Tabela A.1 do Apêndice.

Os escores médios de microinfiltração e os erros-padrão associados, para cada combinação dos níveis dos fatores Material, Campo e Examinador são apresentados na Tabela 1 e representados na Figura 1.

Tabela 1: Escores médios e erros-padrão associados para os dados de microinfiltração para as combinações dos níveis dos fatores Material, Campo e Examinador.

\begin{tabular}{|c|c|c|c|c|}
\hline \multirow[b]{2}{*}{ Material } & \multirow[b]{2}{*}{ Campo } & \multicolumn{3}{|c|}{ Examinador } \\
\hline & & $\mathrm{A}$ & $\mathrm{B}$ & $\mathrm{C}$ \\
\hline \multirow[t]{2}{*}{ Allbond } & Seco & $1.42 \pm 0.31$ & $1.83 \pm 0.24$ & $1.33 \pm 0.31$ \\
\hline & Úmido & $2.00 \pm 0.33$ & $2.17 \pm 0.30$ & $1.58 \pm 0.15$ \\
\hline \multirow[t]{2}{*}{ Optibond } & Seco & $0.92 \pm 0.26$ & $1.50 \pm 0.26$ & $1.08 \pm 0.29$ \\
\hline & Úmido & $0.25 \pm 0.13$ & $0.67 \pm 0.26$ & $0.83 \pm 0.17$ \\
\hline \multirow[t]{2}{*}{ Scotchbond } & Seco & $1.42 \pm 0.34$ & $1.58 \pm 0.34$ & $1.50 \pm 0.26$ \\
\hline & Úmido & $2.67 \pm 0.22$ & $2.92 \pm 0.34$ & $2.08 \pm 0.36$ \\
\hline
\end{tabular}

Por estas medidas podemos notar que para o campo seco, o comportamento do escore médio é similar para as unidades submetidas aos diferentes níveis do fator Material. Já para o campo úmido, o material Optibond parece apresentar melhores resultados, i.e., menores escores de microinfiltração. Nota-se também que o escore médio para o examinador $B$ é maior que o escore médio dos outros examinadores, tanto no campo seco quanto no campo úmido.

Denotaremos por $X_{i j k l}$ o escore atribuído pelo $l$-ésimo examinador, $l=1,2,3$, à su- 
Figura 1: Perfis médios de escore por Material e Campo.
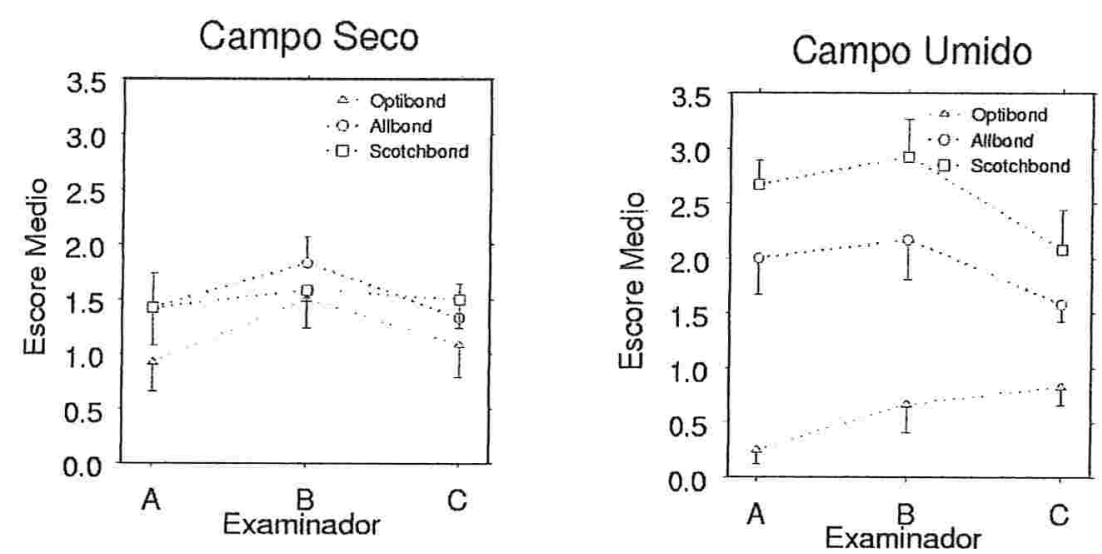

perfície central do $k$-ésimo dente, $k=1,2, \ldots, n_{i j}$, submetido à tratamento com o $i$-ésimo material, $i=1,2,3$ e o $j$-ésimo campo, $j=1,2$. Em ṇotação matricial,

$$
\mathbf{X}_{i j k}=\left(X_{i j k 1}, X_{i j k 2}, X_{i j k 3}\right)^{\prime}
$$

denota as observações associadas ao $k$-ésimo dente submetido ao tratamento correspondente ao $i$-ésimo nível de Material e $j$-ésimo nível de Campo.

Considerando o Escore de microinfiltração e utilizando modelos mistos de Análise de Variância (Crowder and Hand, 1990) para avaliar a influência dos fatores nessa variável resposta, o seguinte modelo paramétrico foi utilizado:

$$
X_{i j k l}=\mu+\alpha_{i}+\beta_{j}+\pi_{k(i j)}+\delta_{l}+\alpha \beta_{i j}+\alpha \delta_{i l}+\beta \delta_{j l}+\alpha \beta \delta_{i j l}+\varepsilon_{i j k l}
$$

com as seguintes restrições de identificabilidade

$$
\begin{aligned}
& \sum_{i=1}^{3} \alpha=\sum_{j=1}^{2} \beta_{j}=\sum_{l=1}^{3} \delta_{l}=0 \\
& \sum_{i=1}^{3} \alpha \beta_{i j}=\sum_{j=1}^{2} \alpha \beta_{i j}=\sum_{i=1}^{3} \alpha \delta_{i l}=\sum_{l=1}^{3} \alpha \delta_{i l}=\sum_{j=1}^{2} \beta \delta_{j l}=\sum_{l=1}^{3} \beta \delta_{j l}=0 \\
& \sum_{i=1}^{3} \alpha \beta \delta_{i j l}=\sum_{j=1}^{2} \alpha \beta \delta_{i j l}=\sum_{l=1}^{3} \alpha \beta \delta_{i j l}=0
\end{aligned}
$$

e $\pi_{k(i j)} \sim N\left(0, \sigma_{\pi}^{2}\right), \varepsilon_{i j k l} \sim N\left(0, \sigma^{2}\right)$ representando variáveis aleatórias independentes.

Nessas condições, 
$\mu$ denota a média geral,

$\alpha_{i}$ denota o efeito principal do $i$-ésimo Material,

$\beta_{j}$ denota o efeito principal de $j$-ésimo Campo,

$\delta_{l}$ denota o efeito principal do $l$-ésimo Examinador,

$\alpha \beta_{i j}$ denota o efeito da interação entre o $i$-ésimo nível de Material e o $j$-ésimo nível de Campo

$\alpha \delta_{i l}$ denota o efeito da interação entre o $i$-ésimo nível de Material e o $l$-ésimo Examinador,

$\beta \delta_{j l}$ denota o efeito da interação entre o $j$-ésimo nível de Campo e o $l$-ésimo Examinador,

$\alpha \beta \delta_{i j l}$ denota o efeito da interação entre o $i$-ésimo nível de Material, o $j$-ésimo nível de Campo e o l-ésimo Examinador.

Como consequência, as variáveis $X_{i j k l}$ têm distribuição Normal com

$$
E\left(X_{i j k l}\right)=\mu+\alpha_{i}+\beta_{j}+\delta_{l}+\alpha \beta_{i j}+\alpha \delta_{i l}+\beta \delta_{j l}+\alpha \beta \delta_{i j l}
$$

e estrutura de covariância definida por

$$
\operatorname{Cov}\left(X_{i j k l}, X_{i^{\prime} j^{\prime} k^{\prime} l^{\prime}}\right)= \begin{cases}\sigma_{\pi}^{2}+\sigma^{2}, & i=i^{\prime}, j=j^{\prime}, k=k^{\prime}, l=l^{\prime} \\ \sigma_{\pi}^{2}, & i=i^{\prime}, j=j^{\prime}, k=k^{\prime}, l \neq l^{\prime} \\ 0, & \text { caso contrário. }\end{cases}
$$

As hipóteses de interesse correspondem à inexistência de efeitos principais e à inexistência de interação entre os fatores.

Na Tabela 2 são apresentados os resultados dos testes das hipóteses usuais da ANOVA para os dados do exemplo, que sugerem um efeito significativo $(\mathrm{p}=0.0012)$ da interação Material x Campo. Modelos reduzidos que incorporam esse comportamento podem ser ajustados para explicar a influência dos fatores na variável resposta (ver Witzel et al., 2000). 
Tabela 2: Estatísticas de teste e níveis descritivos para as hipóteses usuais da ANOVA.

\begin{tabular}{|c|c|c|c|c|}
\hline \multirow[b]{2}{*}{ Fonte } & \multicolumn{2}{|c|}{ Graus de Liberdade } & \multirow[b]{2}{*}{$\mathrm{F}$} & \multirow{2}{*}{$\begin{array}{c}\text { Nível Descritivo } \\
\text { (p-value) }\end{array}$} \\
\hline & Numerador & Denominador & & \\
\hline Material & 2 & 66 & 15.59 & 0.0001 \\
\hline Campo & 1 & 66 & 2.70 & 0.1050 \\
\hline Examinador & 2 & 132 & 5.43 & 0.0054 \\
\hline Material x Campo & 2 & 66 & 7.42 & 0.0012 \\
\hline Material x Examinador & 4 & 132 & 1.44 & 0.2246 \\
\hline Campo x Examinador & 2 & 132 & 0.31 & 0.7369 \\
\hline Material x Campo x & & & & \\
\hline Examinador & 4 & 132 & 1.40 & 0.2382 \\
\hline
\end{tabular}

Como a atribuição de escores às categorias de uma resposta ordenada tem sido objeto de crítica por diversos autores (Ivanova and Berger, 2001, por exemplo), consideramos aqui uma análise de sensibilidade por intermédio da atribuição de outros conjuntos de escores às classes de infiltração: o primeiro $\left(\begin{array}{lllll}0 & 1 & 5 & 9 & 10\end{array}\right)$ considera mais próximas as categorias extremas e o segundo ( 0456610 ) considera mais próximas as categorias intermediárias. Os níveis descritivos para testar as hipóteses usuais são apresentados na Tabela 3.

Tabela 3: Níveis descritivos para as hipóteses da ANOVA.

\begin{tabular}{llccc}
\hline \multicolumn{1}{c}{ Fonte } & & \multicolumn{3}{c}{ Escores } \\
\cline { 1 - 2 } \cline { 5 - 5 } \cline { 5 - 5 } Material & $(01234)$ & $(015910)$ & $(045610)$ \\
Campo & & 0.0001 & 0.0001 & 0.0001 \\
Examinador & & 0.0054 & 0.0693 & 0.2578 \\
Material x Campo & 0.0012 & 0.0048 & 0.0445 \\
Material x Examinador & & 0.2246 & 0.5313 & 0.0005 \\
Campo x Examinador & 0.7369 & 0.6075 & 0.0876 \\
Material x Campo x Examinador & & 0.2382 & 0.4802 & 0.1075 \\
\hline
\end{tabular}

Apesar de as conclusões serem similares, em alguns casos o nível descritivo obtido com os escores modificados chega a ser 8 vezes o nível descritivo original para a mesma hipótese. Por estas razões, um procedimento que utilize somente a ordinalidade da variável resposta pode ser um alternativa bastante atrativa. 


\section{Definição do modelo não-paramétrico e das hipóteses de interesse}

Considere os vetores $\mathbf{X}_{i j k}$ definidos em (1). Sob a hipótese de independência entre as observações de diferentes unidades amostrais, Akritas and Brunner (1997) propõem um modelo não-paramétrico em que a função distribuição de cada elemento $X_{i j k l}$ desşes vetores é a mesma para todas as observações obtidas sob os níveis $i, j$ e $l$ dos fatores envolvidos.

Para contemplar o caso em que a função distribuição de $X_{i j k l}$ não é contínua, utiliza-se sua versão normalizada

$$
F_{i j l}(x)=\frac{1}{2}\left\{F_{i j l}^{+}(x)+F_{i j l}^{-}(x)\right\}
$$

$\operatorname{com} F_{i j l}^{+}(x)=P\left(X_{i j k l} \leq x\right)$ e $F_{i j l}^{-}(x)=P\left(X_{i j k l}<x\right)$, para $i=1,2,3, j=1,2$ e $l=1,2,3$.

O modelo não-paramétrico pode ser considerado como uma generalização do modelo clássico $X_{i j k l} \sim F\left(x-\mu_{i j l}\right), \mu_{i j l}=E\left(X_{i j k l}\right)$, utilizado na seção anterior, sob a suposição adicional que $F$ é Normal com variância $\sigma^{2}$. Nesse contexto, as hipóteses de interesse são baseadas na comparação das médias $\mu_{i j l}$ por meio de combinações lineares que definem os efeitos principais e suas interações.

$\mathrm{Na}$ análise sob o modelo não-paramétrico substituímos as esperanças $\mu_{i j l}$ pelas funções distribuição $F_{i j l}(x)$. Esse enfoque é baseado no conceito de comparação estocástica entre duas ou mais variáveis aleatórias, já abordado em outros procedimentos não-paramétricos, como nos testes propostos por Mann and Whitney (1947) e Kruskall and Wallis (1952).

Como ilustração, considere $Y$ e $Z$ duas variáveis aleatórias contínuas com valores reais e com funções distribuição $F_{y}(y)$ e $F_{z}(z)$, respectivamente. Dizemos que $Y$ é estocasticamente maior que $Z$ se $F_{y}(w)<F_{z}(w)$, para todo $w \in R$. O teste de Mann-Whitney para comparar duas populações utilizando amostras independentes é baseado neste conceito. A hipótese de que as amostras são oriundas da mesma população é formulada como $F_{y}(w)=F_{z}(w)$, para todo $w \in R$.

Para planejamentos mais complexos, com 2 ou mais fatores e com observações correlacionadas, extensões foram formuladas por Akritas and Brunner (1997) em função das 
distribuições definidas em (3), abrangendo os casos em que a variável resposta é medida em escala ordinal.

Seja $\mathbf{F}=\left(F_{111}, F_{112}, \ldots, F_{323}\right)^{\prime}$ o vetor cujo elemento $F_{i j l}$ representa a função distribuição de $X_{i j k l}, i=1,2,3, j=1,2, k=1,2, \ldots, n_{i j}$ e $l=1,2,3$. As hipóteses de interesse são formuladas como combinações lineares de $F_{i j l}$ que podem ser expressas como $\mathrm{CF}=0$, com $\mathrm{C}$ representando uma matriz de contrastes.

Definindo $\mathbf{P}_{a}=\mathbf{I}_{a}-\frac{1}{a} \mathbf{1}_{a} \mathbf{1}_{a}^{\prime}$, com $\mathbf{I}_{a}$ denotando a matriz identidade de ordem $a, \mathbf{1}_{a}$, um vetor com $a$ linhas iguais a $1, \otimes$ representando o produto de Kronecker e

$$
\begin{aligned}
& \bar{F}_{i . .}=\frac{1}{6} \sum_{j=1}^{2} \sum_{l=1}^{3} F_{i j l}, \\
& \bar{F}_{. j .}=\frac{1}{9} \sum_{i=1}^{3} \sum_{l=1}^{3} F_{i j l}, \\
& \bar{F}_{. . l}=\frac{1}{6} \sum_{i=1}^{3} \sum_{j=1}^{2} F_{i j l}, \\
& \bar{F}_{i j .}=\frac{1}{3} \sum_{l=1}^{3} F_{i j l}, \\
& \bar{F}_{i . l}=\frac{1}{2} \sum_{j=1}^{2} F_{i j l}, \\
& \bar{F}_{. j l}=\frac{1}{3} \sum_{i=1}^{3} F_{i j l,}, \\
& \bar{F}_{. . .}=\frac{1}{18} \sum_{i=1}^{3} \sum_{j=1}^{2} \sum_{l=1}^{3} F_{i j l},
\end{aligned}
$$

as hipóteses de inexistência de efeitos principais e de inexistência de interações entre os fatores considerados no exemplo da Seção 1 podem ser explicitadas como

1. Inexistência de efeito principal de Material, $\bar{F}_{i . .}=\bar{F}_{\ldots,}, i=1,2,3$, que corresponde a $\mathrm{CF}=0, \operatorname{com} \mathrm{C}=\mathrm{C}_{\mathrm{M}}=\left(\mathbf{P}_{\mathbf{3}} \otimes \frac{1}{2} \mathbf{1}_{\mathbf{2}}^{\prime} \otimes \frac{1}{3} \mathbf{1}_{\mathbf{3}}^{\prime}\right)$.

2. Inexistência de efeito principal de Campo, $\bar{F}_{. j .}=\bar{F}_{\ldots,}, j=1,2$, que corresponde a $\mathrm{CF}=0, \operatorname{com} \mathrm{C}=\mathrm{C}_{\mathrm{C}}=\left(\frac{1}{3} \mathbf{1}_{3}^{\prime} \otimes \mathbf{P}_{2} \otimes \frac{1}{3} \mathbf{1}_{3}^{\prime}\right)$.

3. Inexistência de efeito principal de Examinador, $\bar{F}_{. . l}=\bar{F}_{\ldots}, l=1,2,3$, que corresponde a $\mathrm{CF}=0$, com $\mathrm{C}=\mathrm{C}_{\mathrm{E}}=\left(\frac{1}{3} \mathbf{1}_{3}^{\prime} \otimes \frac{1}{2} \mathbf{1}_{2}^{\prime} \otimes \mathrm{P}_{3}\right)$. 
4. Inexistência de interação Material x Campo, $\bar{F}_{i j .}=\bar{F}_{i . .}+\bar{F}_{. j .}-\bar{F}_{\ldots}, \quad i=1,2,3$, $j=1,2$, que corresponde a $\mathbf{C F}=0$, com $\mathbf{C}=\mathbf{C}_{\mathbf{M C}}=\left(\mathbf{P}_{\mathbf{3}} \otimes \mathbf{P}_{\mathbf{2}} \otimes \frac{1}{3} \mathbf{1}_{\mathbf{3}}^{\prime}\right)$.

5. Inexistência de interação Material x Examinador, $\bar{F}_{i . l}=\bar{F}_{i . .}+\bar{F}_{. . l}-\bar{F}_{\ldots,}, \quad i=1,2,3$, $l=1,2,3$, que corresponde a $\mathbf{C F}=0$, com $\mathbf{C}=\mathbf{C}_{\mathrm{ME}}=\left(\mathbf{P}_{\mathbf{3}} \otimes \frac{1}{2} \mathbf{1}_{\mathbf{2}}^{\prime} \otimes \mathbf{P}_{\mathbf{3}}\right)$.

6. Inexistência de interação Campo x Examinador, $\bar{F}_{. j l}=\bar{F}_{. j .}+\bar{F}_{. . l}-\bar{F}_{. .}, \quad j=1,2$, $l=1,2,3$, que corresponde a $\mathbf{C F}=0$, com $\mathbf{C}=\mathbf{C}_{\mathbf{C E}}=\left(\frac{1}{3} \mathbf{1}_{3}^{\prime} \otimes \mathbf{P}_{\mathbf{2}} \otimes \mathbf{P}_{3}\right)$.

7. Inexistência de interação Material x Campo x Examinador, $\bar{F}_{i j l}=\bar{F}_{\text {.. }}-\bar{F} i$.. $\bar{F} . j .-\bar{F}_{. . l}+\bar{F}_{i j .}+\bar{F}_{i . l}+\bar{F}_{. j l}, \quad i=1,2,3, \quad j=1,2, \quad l=1,2,3$, que corresponde a $\mathrm{CF}=0, \operatorname{com} \mathrm{C}=\mathrm{C}_{\mathrm{MCE}}=\left(\mathbf{P}_{\mathbf{3}} \otimes \mathbf{P}_{\mathbf{2}} \otimes \mathbf{P}_{3}\right)$.

A representação das hipóteses é muito semelhante àquela utilizada no modelo clássico. Para interpretar essas hipóteses, considere as duas variáveis aleatórias $Y$ e $Z$ definidas anteriormente e seja $p=P(Y \leq Z)=\int F_{z} d F_{y}$. Sob a hipótese $F_{y}(w)=F_{z}(w)$, para todo $w \in R, p=1 / 2$. Já se $Y$ é estocasticamente maior (menor) que $Z$ essa quantidade deve ser menor (maior) que $1 / 2$. A probabilidade $p$ descreve o efeito (não-paramétrico) de tratamento que é generalizado para o caso de variáveis ordinais com medidas repetidas por intermédio das probabilidades $p_{i j l}=\int H d F_{i j l}, i=1,2,3, j=1,2$ e $l=1,2,3$, com $H(x)=N^{-1} \sum_{i=1}^{3} \sum_{j=1}^{2} \sum_{l=1}^{3} n_{i j} F_{i j l}(x), N=\sum_{i=1}^{3} \sum_{j=1}^{2} \sum_{l=1}^{3} n_{i j}$.

A quantidade $p_{i j l}$ é denominada efeito relativo de tratamento associado ao nível $i$ do fator Material, $j$ do fator Campo e $l$ do fator Examinador com respeito à $H$, a média ponderada das funções de distribuição marginais de $X_{i j k l}$. Ela pode ser interpretada como a probabilidade de uma observação selecionada ao acaso do tratamento representado pelos níveis $i, j$ e $l$ dos fatores envolvidos ser classificada numa categoria de ordem maior que uma observação selecionada aleatoriamente de todo o conjunto.

Note que podemos escrever o vetor $\mathbf{p}=\left(p_{111}, p_{112}, p_{113}, \ldots, p_{321}, p_{322}, p_{323}\right)^{\prime}$ em função do vetor $\mathbf{F}$, como $\mathbf{p}=\int H d \mathbf{F}$. Para as matrizes de contrastes definidas anteriormente, $\mathrm{Cp}=\mathrm{C} \int H d \mathbf{F}=\int H d \mathbf{C F}$. Desse modo, a hipótese $\mathbf{C F}=0$ implica $\mathbf{C p}=0$ e as interpretações correspondentes podem ser baseadas nos efeitos relativos $p_{i j l}$, ou seja,

1. Inexistência de efeito principal de Material

$$
\bar{F}_{i . .}=\bar{F}_{\ldots .} \Rightarrow \bar{p}_{i . .}=\bar{p}_{\ldots}
$$


2. Inexistência de efeito principal de Campo

$$
\bar{F}_{. j .}=\bar{F}_{\ldots .} \Rightarrow \bar{p}_{. j .}=\bar{p}_{\ldots}
$$

3. Inexistência de efeito principal de Examinador

$$
\bar{F}_{. . l}=\bar{F}_{. .} \Rightarrow \bar{p}_{. . l}=\bar{p}_{\ldots}
$$

4. Inexistência de interação Material x Campo

$$
\bar{F}_{i j .}=\bar{F}_{i . .}+\bar{F}_{. j .}-\bar{F}_{\ldots .} \Rightarrow \bar{p}_{i j .}=\bar{p}_{i . .}+\bar{p}_{. j .}-\bar{p}_{\ldots}
$$

5. Inexistência de interação Material x Examinador

$$
\bar{F}_{i . l}=\bar{F}_{i . .}+\bar{F}_{. . l}-\bar{F}_{\ldots .} \Rightarrow \bar{p}_{i . l}=\bar{p}_{i . .}+\bar{p}_{. . l}-\bar{p}_{\ldots .}
$$

6. Inexistência de interação Campo x Examinador

$$
\bar{F}_{. j l}=\bar{F}_{. j .}+\bar{F}_{. . l}-\bar{F}_{. . .} \Rightarrow \bar{p}_{. j l}=\bar{p}_{. j .}+\bar{p}_{. . l}-\bar{p}_{. .}
$$

7. Inexistência de interação Material x Campo x Examinador

$$
\begin{aligned}
\bar{F}_{i j l} & =\bar{F}_{. .}-\bar{F}_{i . .}-\bar{F}_{. j .}-\bar{F}_{. . l}+\bar{F}_{i j .}+\bar{F}_{i . l}+\bar{F}_{. j l} \Rightarrow \\
\bar{p}_{i j l} & =\bar{p}_{. .}-\bar{p}_{i . .}-\bar{p}_{. j .}-\bar{p}_{. . l}+\bar{p}_{i j .}+\bar{p}_{i . l}+\bar{p}_{. j l},
\end{aligned}
$$

com

$$
\begin{aligned}
& \bar{p}_{i . .}=\frac{1}{6} \sum_{j=1}^{2} \sum_{l=1}^{3} p_{i j l} \\
& \bar{p}_{. j .}=\frac{1}{9} \sum_{i=1}^{3} \sum_{l=1}^{3} p_{i j l} \\
& \bar{p}_{. . l}=\frac{1}{6} \sum_{i=1}^{3} \sum_{j=1}^{2} p_{i j l} \\
& \bar{p}_{i j .}=\frac{1}{3} \sum_{l=1}^{3} p_{i j l} \\
& \bar{p}_{i . l}=\frac{1}{2} \sum_{j=1}^{2} p_{i j l}
\end{aligned}
$$




$$
\begin{aligned}
& \bar{p}_{. j l}=\frac{1}{3} \sum_{i=1}^{3} p_{i j l} \\
& \bar{p}_{. \ldots}=\frac{1}{18} \sum_{i=1}^{3} \sum_{j=1}^{2} \sum_{l=1}^{3} p_{i j l}
\end{aligned}
$$

Para exemplificar, considere a hipótese de inexistência de efeito de Campo. Dado que quanto maior o escore, pior o nível de microinfiltração no dente, se o campo úmido for mais susceptível à microinfiltração, os escores associados às unidades desse grupo $(j=2)$ tendem a ser maiores que aqueles associados às unidades do grupo submetido ao tratamento com campo seco $(j=1)$. Assim, $F_{.2 .}<F_{.1}$ e a hipótese $F_{.1}=F_{.2}$ deve ser rejeitada. Isso implica $p_{.2 .}>p_{.1}$, que indica que o efeito relativo para o campo úmido é maior que o efeito relativo para o campo seco. As interpretações para os outros efeitos são análogas.

\section{Estimação dos efeitos dos tratamentos e estatísticas de teste}

Para estimar os efeitos dos tratamentos, $p_{i j l}$, definidos na seção anterior, utilizamos a função distribuição empírica normalizada $\hat{F}_{i j l}(x)$ das variáveis $X_{i j k l}$, nomeadamente

$$
\hat{F}_{i j l}(x)=n_{i j}^{-1} \sum_{k=1}^{n_{i j}} \frac{1}{2}\left\{1_{\left\{X_{i j k l<x\}}\right.}+1_{\left\{X_{i j k l \leq x}\right\}}\right\}
$$

onde $1_{\{.\}}$denota a função indicadora.

A função $H(x)$ é estimada por

$$
\hat{H}(x)=N^{-1} \sum_{i=1}^{3} \sum_{j=1}^{2} \sum_{l}^{3} n_{i j} \hat{F}_{i j l}(x) .
$$

Para estimar $p_{i j l}$ utilizamos

$$
\hat{p}_{i j l}=\int \hat{H} d \hat{F}_{i j l}=\sum_{k=1}^{n_{i j}} \hat{H}\left(X_{i j k l}\right) \cdot P\left(X_{i j k l}=x_{i j k l}\right)=n_{i j}^{-1} \sum_{k=1}^{n_{i j}} \hat{H}\left(X_{i j k l}\right)
$$

$\operatorname{com} \hat{H}\left(X_{i j k l}\right)=N^{-1}\left(R_{i j k l}-1 / 2\right), R_{i j k l}$ denotando o posto da observação $X_{i j k l}$ dentre todas as observações da amostra. Desse modo, os estimadores dos efeitos de tratamento são dados por

$$
\hat{p}_{i j l}=n_{i j}^{-1} \sum_{k=1}^{n_{i j}} N^{-1}\left(R_{i j k l}-1 / 2\right)=N^{-1}\left(\bar{R}_{i j . l}-1 / 2\right)
$$


com $\bar{R}_{i j . l}$ denotando a média dos postos das $n_{i j}$ observações coletadas em unidades submetidas ao tratamento correspondente aos níveis $i, j$ e $l$ dos fatores Material, Campo e Examinador, respectivamente. Podemos então representar os efeitos estimados pelo vetor $\hat{\mathbf{p}}=\left(\hat{\mathbf{p}}_{11}^{\prime}, \hat{\mathbf{p}}_{12}^{\prime}, \ldots, \hat{\mathbf{p}}_{32}^{\prime}\right)^{\prime}$ com $\hat{\mathbf{p}}_{i j}=\left(\hat{p}_{i j 1}, \hat{p}_{i j 2}, \hat{p}_{i j 3}\right)^{\prime}$. Este estimador é consistente para $\mathrm{p}$, conforme demonstram Akritas and Brunner (1997).

Para os dados do exemplo, apresentamos as estimativas dos efeitos relativos de tratamento na Tabela 4 e sua representação gráfica na Figura 2.

Tabela 4: Estimativas dos efeitos relativos de tratamento para os dados de microinfiltração.

\begin{tabular}{|c|c|c|c|c|}
\hline \multirow[b]{2}{*}{ Material } & \multirow[b]{2}{*}{ Campo } & \multicolumn{3}{|c|}{ Examinador } \\
\hline & & $\mathrm{A}$ & B & $\mathrm{C}$ \\
\hline \multirow[t]{2}{*}{ Allbond } & Seco & 0.48 & 0.59 & 0.45 \\
\hline & Úmido & 0.61 & 0.66 & 0.53 \\
\hline \multirow[t]{2}{*}{ Optibond } & Seco & 0.34 & 0.50 & 0.37 \\
\hline & Úmido & 0.17 & 0.28 & 0.32 \\
\hline \multirow[t]{2}{*}{ Scotchbond } & Seco & 0.47 & 0.52 & 0.50 \\
\hline & Úmido & 0.78 & 0.80 & 0.63 \\
\hline
\end{tabular}

Figura 2: Efeitos relativos de tratamento para os dados do estudo de microinfiltração.
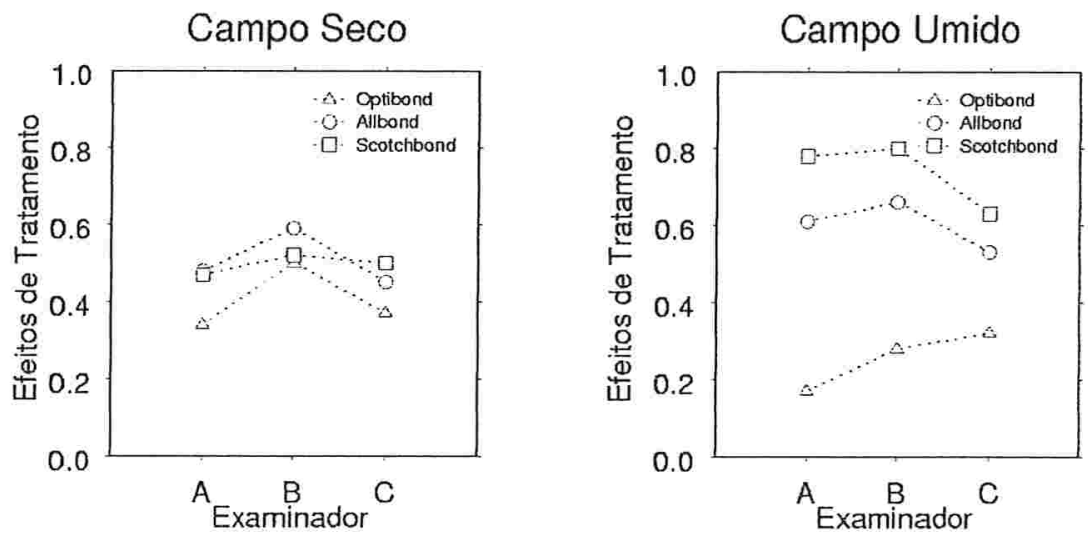

O comportamento dos efeitos de tratamentos observado nesses gráficos é semelhante ao comportamento dos escores médios representados na Figura 1. O material Optibond 
apresenta os menores efeitos quanto à microinfiltração em campo úmido e o examinador $B$ parece avaliar a microinfiltração mais severamente que os demais observadores.

\subsection{Distribuição dos estimadores dos efeitos de tratamento}

Os resultados utilizados para testar as hipóteses de interesse são baseados na distribuição assintótica de $\sqrt{n} \hat{\mathbf{p}}$, com $n=\sum_{i=1}^{3} \sum_{j=1}^{2} n_{i j}$ denotando o número de unidades experimentais. Note que $\sqrt{n} \hat{\mathbf{p}}$ pode ser escrito como $(\sqrt{n} / N)\left(\overline{\mathbf{R}}-\frac{1}{2} \mathbf{1}_{18}\right), \overline{\mathbf{R}}=\left(\bar{R}_{11.1}, \bar{R}_{11.2}, \ldots, \bar{R}_{32.3}\right)$, $\bar{R}_{i j . l}=\left(n_{i j}^{-1} \sum_{k=1}^{n_{i j}} R_{i j k l}\right)$. Então, para obter a distribuição de $\sqrt{n} \hat{\mathbf{p}}$ pode-se utilizar a distribuição dos vetores $\sqrt{n} / N \overline{\mathbf{R}}_{i j}=\left(\bar{R}_{i j .1}, \bar{R}_{i j .2}, \bar{R}_{i j .3}\right)^{\prime}, i=1,2,3, j=1,2$.

Como os postos $R_{i j k l}$ são calculados com base em todas as $N$ observações, esses vetores não são independentes e o Teorema Limite Central na sua versão usual não pode ser aplicado diretamente. A solução é encontrar outros vetores que tenham, assintoticamente, a mesma distribuição de $(\sqrt{n} / N) \overline{\mathbf{R}}$ e que possam ser escritos como médias de vetores aleatórios independentes.

Sejam $\mathbf{X}_{i j k}=\left(X_{i j k 1}, X_{i j k 2}, X_{i j k 3}\right)^{\prime}$ os vetores definidos em $(1), \hat{H}(x), H(x), \hat{p}_{i j l}, p_{i j l} \mathrm{e}$ $F_{i j l}$ definidos nas Seções 3 e 4 e considere $W_{i j l}=\left(\hat{p}_{i j l}-\int \hat{H} d F_{i j l}\right)$. Akritas and Brunner (1997) demostram que, para $n \rightarrow \infty$, com $n / n_{i j} \leq n_{0} \leq \infty, W_{i j l}$ é assintoticamente equivalente a $U_{i j l}=\sqrt{n} / n_{i j} \sum_{k=1}^{n_{i j}}\left(H\left(X_{i j k l}\right)-p_{i j l}\right), i=1,2,3, j=1,2, l=1,2,3$, ou seja, que a diferença entre $\left(W_{i j l}-U_{i j l}\right)$ tende a zero em probabilidade e conseqüentemente, que as variáveis $W_{i j l}$ e $U_{i j l}$ têm a mesma distribuição assintótica.

Os vetores $H\left(\mathbf{X}_{i j k}\right)=\left(H\left(X_{i j k 1}\right), H\left(X_{i j k 2}\right), H\left(X_{i j k 3}\right)\right)^{\prime}$ são independentes para $(i, j, k) \neq$ $\left(i^{\prime}, j^{\prime}, k^{\prime}\right)$ uma vez que $\mathbf{X}_{i j k}$ são independentes e a transformação $H($.$) é uma média pon-$ derada das funções de distribuição marginais $F_{i j l}$. Desse modo, $\bar{H}_{i j .}=\left(\bar{H}_{i j 1}, \bar{H}_{i j 2}, \bar{H}_{i j 3}\right)^{\prime}$ são vetores de médias de variáveis aleatórias independentes com $\bar{H}_{i j l}=n_{i j}^{-1} \sum_{k=1}^{n_{i j}} H\left(X_{i j k l}\right)$ e pelo Teorema Limite Central possuem assintoticamente distribuição normal.

Da equivalência assintótica entre as variáveis $W_{i j l}$ e $U_{i j l}$ decorre que as quantidades $\sqrt{n} \hat{\mathbf{p}}$ e $\sqrt{n}\left(\overline{\mathbf{H}}+\int \hat{H} d \mathbf{F}-\mathbf{p}\right)$ são assintoticamente equivalentes, com $\overline{\mathbf{H}}=\left(\bar{H}_{11}^{\prime}, \ldots, \bar{H}_{32}^{\prime}.\right)$.

Para testar as hipoteses de interesse é necessário obter a distribuição de $\sqrt{n} \mathbf{C} \hat{\mathbf{p}}$ sob a hipótese $\mathbf{C p}=0$. Mas, $\mathbf{C p}=0$ implica $\mathbf{C F}=0$ e $\sqrt{n}\left(\mathbf{C H}+\mathbf{C} \int \hat{H} d \mathbf{F}-\mathbf{C p}\right)=\sqrt{n} \mathbf{C} \overline{\mathbf{H}}$. Portanto sob as hipóteses de interesse, $\sqrt{n} \mathrm{C} \hat{\mathrm{p}}$ e $\sqrt{n} \mathrm{CH}$ são equivalentes assintoticamente, ou seja, $\sqrt{n} \mathbf{C} \hat{\mathbf{p}}$ tem distribuição assintoticamente normal, com $E(\sqrt{n} \mathbf{C} \hat{\mathbf{p}})=E(\sqrt{n} \mathbf{C} \overline{\mathbf{H}})=$ 
0 e $\operatorname{Var}(\sqrt{n} \mathbf{C} \hat{\mathbf{p}})=\operatorname{Var}(\sqrt{n} \mathbf{C H})$.

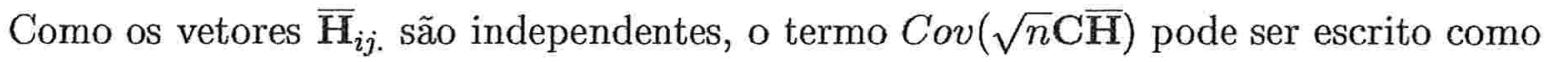
$\mathrm{CVC}^{\prime} \operatorname{com} \mathbf{V}=\operatorname{Cov}(\sqrt{n} \overline{\mathbf{H}})=n \operatorname{diag}\left\{n_{11}^{-1} \mathbf{V}_{\mathbf{1 1}}, n_{12}^{-1} \mathbf{V}_{\mathbf{1 2}}, \ldots, n_{32}^{-1} \mathbf{V}_{\mathbf{3 2}}\right\}$ e $\mathbf{V}_{i j}=\operatorname{Cov}\left(H\left(\mathbf{X}_{i j k}\right)\right)$.

\subsection{Estatísticas de Teste}

Apresentaremos nesta seção dois procedimentos para testar a hipótese $\mathbf{C F}=\mathbf{0}$, ambos baseados em modificações da estatística de Wald para postos (ver Brunner and Langer, 2000). Primeiramente consideremos o estimador

$$
\hat{\mathbf{V}}=\bigoplus_{i=1}^{3} \bigoplus_{j=1}^{2} \frac{n}{n_{i j}} \hat{\mathbf{V}}_{i j}
$$

com

$$
\hat{\mathbf{V}}_{i j}=\frac{1}{N^{2}\left(n_{i j}-1\right)} \sum_{k=1}^{n_{i j}}\left(\mathbf{R}_{i j k}-\overline{\mathbf{R}}_{i j . .}\right)\left(\mathbf{R}_{i j k}-\overline{\mathbf{R}}_{i j . .}\right)^{\prime}, \quad i=1,2,3 \quad j=1,2
$$

que é consistente para V conforme demostram Akritas and Brunner (1997).

Sob as hipóteses utilizadas para derivar a distribuição assintótica de $\sqrt{n} \mathbf{C} \hat{\mathbf{p}}$ na subseção anterior, a estatística $Q_{W}(\mathbf{C})=n \hat{\mathbf{p}}^{\prime} \mathbf{C}^{\prime}\left[\mathbf{C} \hat{\mathbf{V}} \mathbf{C}^{\prime}\right]-\mathbf{C} \hat{\mathbf{p}}$, tem distribuição assintótica $\chi_{f}^{2}$ central $\operatorname{com} f=\operatorname{posto}(\mathbf{C})$. A notação $\left[\mathbf{C V}^{\prime}\right]^{-}$indica uma inversa generalizada de $[\mathbf{C} \hat{\mathbf{V}} \mathbf{C}]$.

Utilizando estudos de simulação, Brunner and Langer (2000) e Munzel and Brunner (2000) discutiram a aproximação à distribuição assintótica da estatística $Q_{W}(\mathrm{C})$ para amostras pequenas, notando que a convergência para a distribuição Qui-quadrado é bastante lenta e, consequentemente que os níveis descritivos para os testes são bastante liberais. A quantidade de parâmetros da matriz de covariâncias V que necessitam ser estimados é um dos fatores que afetam a qualidade deste teste. Brunner and Langer (2000) propuseram uma estatística alternativa, baseada numa correção para a estatistica $Q_{W}$. Esta estatística é dada por $Q_{A}(\mathbf{C})=n \hat{\mathbf{p}}^{\prime} \mathbf{M} \hat{\mathbf{p}} / \operatorname{tr}(\mathbf{M} \hat{\mathbf{V}}), \operatorname{com} \mathbf{M}=\mathbf{C}^{\prime}\left[\mathbf{C C}^{\prime}\right]^{-} \mathbf{C}$. Sob as hipóteses apresentadas na seção anterior, Brunner et al. (1999) demonstram que essa estatística tem distribuição aproximadamente $\chi_{\hat{f}}^{2}$, com

$$
\hat{f}=[\operatorname{tr}(\mathbf{M} \hat{\mathbf{V}})]^{2} / \operatorname{tr}(\mathbf{M} \hat{\mathbf{V}} \mathbf{M} \hat{\mathbf{V}})
$$


para $\operatorname{tr}(\mathbf{M} \hat{\mathbf{V}})>0$. A convergência da distribuição dessa estatística para sua distribuição assintótica é mais rápida do que a da estatística $Q_{w}(C)$. Esse resultado é útil em situações onde o tamanho da amostra é pequeno.

\section{Comparação entre os Resultados}

Para testar as hipóteses apresentadas na Seção 3 no caso especial do estudo de microinfiltração, utilizamos as duas estatísticas definidas na seção anterior. Os resultados estão dispostos na Tabela 5. Por estes resultados, somente a interação Material x Campo foi significativa, evidenciando o mesmo comportamento observado quando utilizamos o modelo paramétrico.

Tabela 5: Estatísticas de teste e níveis descritivos para as hipóteses usuais.

\begin{tabular}{|c|c|c|c|c|}
\hline \multirow[b]{2}{*}{ Fonte } & \multicolumn{2}{|c|}{$Q_{W}$} & \multicolumn{2}{|c|}{$Q_{A}$} \\
\hline & $\begin{array}{c}\text { Graus } \\
\text { de } \\
\text { liberdade }\end{array}$ & $\begin{array}{c}\text { Nível } \\
\text { Descritivo } \\
\text { (p-value) }\end{array}$ & $\begin{array}{c}\text { Graus } \\
\text { de } \\
\text { liberdade }\end{array}$ & $\begin{array}{c}\text { Nível } \\
\text { Descritivo } \\
\text { (p-value) }\end{array}$ \\
\hline Material & 2 & 0.0000 & 1.96 & 0.0000 \\
\hline Campo & 1 & 0.1432 & 1.00 & 0.1432 \\
\hline Examinador & 2 & 0.0090 & 1.88 & 0.0057 \\
\hline Material x Campo & 2 & 0.0010 & 1.96 & 0.0011 \\
\hline Material x Examinador & 4 & 0.3336 & 3.47 & 0.2401 \\
\hline Campo x Examinador & 2 & 0.6843 & 1.88 & 0.7379 \\
\hline Material x Campo x & & & & \\
\hline Examinador & 4 & 0.1512 & 3.47 & 0.2321 \\
\hline
\end{tabular}

Como no caso paramétrico, modelos reduzidos podem ser ajustados aos dados. Considerando, por exemplo, um modelo contendo os efeitos principais dos fatores Material, Campo e Examinador, e o efeito de interação dos fatores Material e Campo, as novas estimativas dos efeitos relativos de tratamento são dadas na Tabela 6 e são mostradas na Figura 3.

A comparação dos efeitos relativos de tratamento para os escores atribuídos pelos examinadores $A$ e $C$ sugere que um modelo ainda mais restrito, que não considere diferenças entre as classificações destes examinadores pode ser ajustado. As estimativas para 
Tabela 6: Estimativas dos efeitos relativos de tratamento para os dados de microinfiltração sob o modelo reduzido.

\begin{tabular}{lcccccc}
\hline \multirow{2}{*}{ Material } & & & & \multicolumn{3}{c}{ Examinador } \\
\cline { 1 - 1 } \cline { 5 - 6 } Allbond & & Seco & & 0.48 & 0.56 & 0.47 \\
\cline { 5 - 6 } Optibond & & Úmido & & 0.58 & 0.66 & 0.47 \\
& & Seco & & 0.38 & 0.46 & 0.37 \\
Scotchbond & & Úmido & & 0.23 & 0.31 & 0.22 \\
& & Seco & & 0.47 & 0.55 & 0.46 \\
& & Úmido & & 0.71 & 0.79 & 0.70 \\
\hline
\end{tabular}

Figura 3: Efeitos relativos de tratamento estimados sob o modelo reduzido.
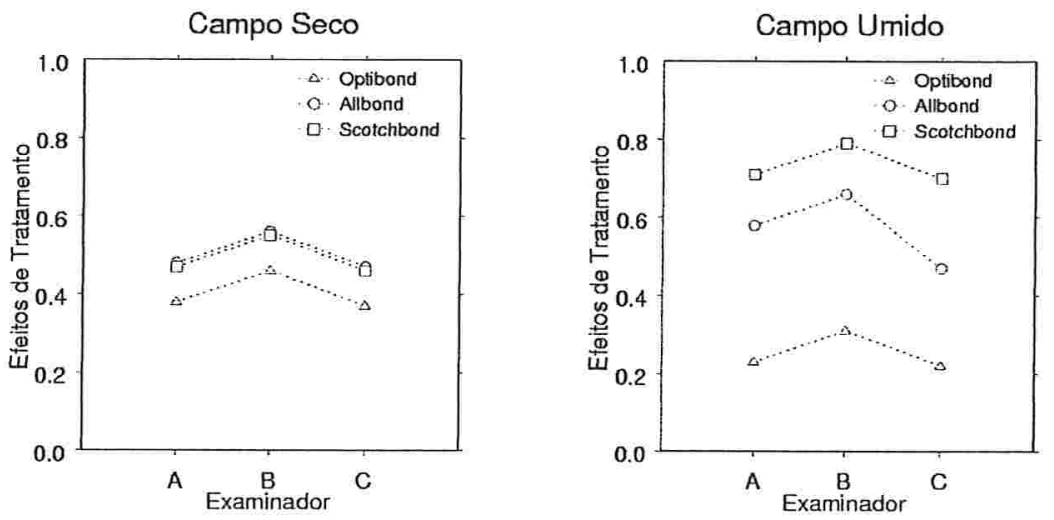

os efeitos relativos de tratamento sob esse novo modelo são dadas na Tabela 7.

Tabela 7: Estimativas dos efeitos relativos de tratamento sob o modelo que não considera diferença entre os efeitos relativos de tratamento para os Examinadores $A$ e $C$.

\begin{tabular}{lcccccc}
\hline \multirow{2}{*}{ Material } & & & & \multicolumn{3}{c}{ Examinador } \\
\cline { 1 - 1 } \cline { 5 - 7 } Allbond & & Seco & & 0.48 & 0.56 & 0.48 \\
& & Úmido & & 0.57 & 0.66 & 0.57 \\
Optibond & & Seco & & 0.37 & 0.46 & 0.37 \\
& & Úmido & & 0.23 & 0.31 & 0.23 \\
Scotchbond & & Seco & & 0.47 & 0.55 & 0.47 \\
& & Úmido & & 0.71 & 0.79 & 0.71 \\
\hline
\end{tabular}




\section{Simulação}

Apresentamos nesta seção as conclusões de um estudo de simulação cujo objetivo é comparar os resultados obtidos sob o modelo paramétrico para escores médios de resposta e sob o modelo não-paramétrico correspondente. Estudamos o comportamento das estatísticas de teste apresentadas na Seção 4 em duas situações. Na primeira construímos conjuntos de dados com o mesmo planejamento do estudo de microinfiltração apresentado na Seção 1. Utilizamos o mesmo número de fatores, de níveis e de observações por tratamento. Para esses dados, comparamos as taxas de erro Tipo-I obtidas segundo os diferentes procedimentos de análise.

Dada a complexidade de simulação sob o modelo do Exemplo 1, estudamos o poder dos testes mencionados nas Seções 2 e 4 utilizando amostras obtidas sob um modelo mais simples, com três estratos e duas medidas por unidade experimental.

\subsection{Comparação das taxas de erro Tipo-I}

Para esse finalidade, geramos 1000 amostras de vetores independentes

$$
\mathbf{X}_{i j k}=\left(X_{i j k 1}, X_{i j k 2}, X_{i j k 3}\right)^{\prime}
$$

$i=1,2,3, j=1,2$ e $k=1,2, \ldots, n$ com

$$
X_{i j k l}=\operatorname{int}\left[5 * \frac{\left(c * Z_{i j k}\right)+Y_{i j k l}}{c+1}\right]
$$

e com $Z_{i j k}$ e $Y_{i j k l}$ independentes e com distribuição $U(0,1), c>0$ uma constante e $\operatorname{int}(x)$ denotando o maior número inteiro menor que $x$. Os componentes do vetor assim construído assumem valores de 0 a 4 e são correlacionados. A correlação entre $X_{i j k l}$ e $X_{i j k l^{\prime}}$ depende da escolha da constante $c$, conforme pode ser observado na Figura 4. Maiores valores de $c$ implicam maior correlação entre esses componentes.

Essa mesma proposta foi utilizada por Brunner and Langer (2000) para estudar a distribuição assintótica da estatística tipo-ANOVA apresentada na Seção 4. Segundo 
Figura 4: Correlação de Spearman em função da constante c.

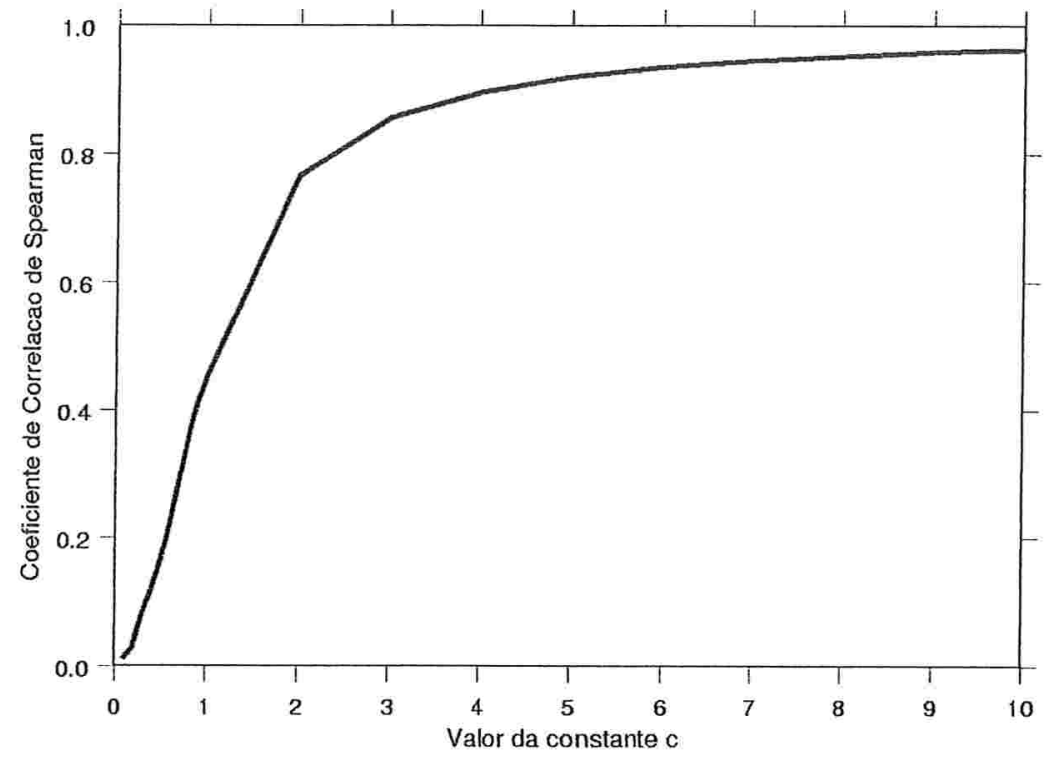

esses autores, valores diferentes de $c$ resultaram em conclusões similares. Adotamos para nosso estudo o valor $c=1$.

De acordo com a definição de $X_{i j k l}$, as hipóteses nulas especificadas na Seção 3 são todas válidas. Para estudarmos o efeito da atribuição de escores nos resultados, utilizamos 3 esquemas de pontuação das classes de resposta, o primeiro com escores igualmente espaçados (0 1234 ), o segundo com escores dados por (0 159 10), que considera mais próximas as categorias extremas de resposta e o último, (0 456 10), que considera mais próximas as categorias intermediárias de resposta.

As taxas de erro do Tipo-I calculadas a partir dos resultados dos testes são apresentadas nas Tabelas 8 e 9.

Os testes não-paramétricos, em geral, apresentaram taxas de erro maiores que as obtidas com os testes paramétricos. Para a taxa nominal $\alpha=1 \%$, as taxas de erro dos testes paramétricos diferem do valor esperado por não mais de $60 \%$, enquanto a mesma diferença para as taxas associadas aos testes não-paramétricos alcança 120\% com a estatística de Wald e 60\% com a estatística tipo-ANOVA. Para $\alpha=5 \%$, as taxas diferem do esperado por, no máximo, $22 \%$ para os testes paramétricos, $60 \%$ para o teste 
Tabela 8: Taxas de erro Tipo-I baseadas em amostras geradas por (13) para testes das hipóteses usuais - Nível de Significância de $1 \%$.

\begin{tabular}{|c|c|c|c|c|c|}
\hline \multirow[b]{2}{*}{ Fonte } & \multicolumn{3}{|c|}{$\begin{array}{c}\text { Testes paramétricos com } \\
\text { Escores }\end{array}$} & \multicolumn{2}{|c|}{$\begin{array}{c}\text { Testes não } \\
\text { Paramétricos }\end{array}$} \\
\hline & $\left(\begin{array}{lllll}0 & 1 & 2 & 3 & 4\end{array}\right)$ & $\left(\begin{array}{lllll}0 & 1 & 5 & 9 & 10\end{array}\right)$ & $(045610)$ & Wald & ANOVA \\
\hline Material & 0.010 & 0.005 & 0.013 & 0.018 & 0.011 \\
\hline Campo & 0.011 & 0.013 & 0.011 & 0.015 & 0.015 \\
\hline Examinador & 0.009 & 0.009 & 0.012 & 0.017 & 0.008 \\
\hline Material x Campo & 0.009 & 0.010 & 0.007 & 0.017 & 0.016 \\
\hline Material x Examinador & 0.014 & 0.013 & 0.012 & 0.022 & 0.012 \\
\hline Campo x Examinador & 0.011 & 0.010 & 0.009 & 0.016 & 0.011 \\
\hline Material x Campo x & & & & & \\
\hline Examinador & 0.006 & 0.004 & 0.006 & 0.022 & 0.007 \\
\hline
\end{tabular}

Tabela 9: Taxas de erro Tipo-I baseadas em amostras geradas por (13) para testes das hipóteses usuais - Nível de Significância de $5 \%$.

\begin{tabular}{|c|c|c|c|c|c|}
\hline \multirow[b]{2}{*}{ Fonte } & \multicolumn{3}{|c|}{$\begin{array}{c}\text { Testes paramétricos com } \\
\text { Escores }\end{array}$} & \multicolumn{2}{|c|}{$\begin{array}{c}\text { Testes não } \\
\text { Paramétricos }\end{array}$} \\
\hline & $\left(\begin{array}{lllll}0 & 1 & 2 & 3 & 4\end{array}\right)$ & $\left(\begin{array}{lllll}0 & 1 & 5 & 9 & 10\end{array}\right)$ & $(0445610)$ & Wald & ANOVA \\
\hline Material & 0.059 & 0.056 & 0.050 & 0.073 & 0.065 \\
\hline Campo & 0.054 & 0.047 & 0.051 & 0.055 & 0.055 \\
\hline Examinador & 0.057 & 0.059 & 0.046 & 0.064 & 0.060 \\
\hline Material x Campo & 0.044 & 0.045 & 0.049 & 0.053 & 0.050 \\
\hline Material x Examinador & 0.047 & 0.046 & 0.053 & 0.080 & 0.047 \\
\hline Campo x Examinador & 0.049 & 0.048 & 0.048 & 0.062 & 0.052 \\
\hline Material x Campo x & & & & & \\
\hline Examinador & 0.039 & 0.048 & 0.043 & 0.070 & 0.047 \\
\hline
\end{tabular}

não-paramétrico de Wald e 30\% para o teste tipo-ANOVA.

Nota-se que os níveis descritivos obtidos com a estatística de Wald são mais liberais que aqueles obtidos com a estatística tipo-ANOVA que, por sua vez se apresentam mais próximos dos níveis obtidos com a análise paramétrica.

\subsection{Comparação do Poder dos Testes}

Nesta seção apresentaremos os resultados da análise de 1000 amostras geradas sob um planejamento com 2 fatores cruzados. O primeiro $(G)$, com 3 níveis, é um fator entre 
unidades amostrais e o segundo $(R)$, com 2 níveis, é um fator intra unidades amostrais. $\mathrm{O}$ interesse é estudar o poder dos testes não-paramétricos comparando-os com o poder dos testes obtidos com a análise de variância usual para os escores. As observações simuladas são representadas por $\mathbf{X}_{i k}=\left(X_{i k 1}, X_{i k 2}\right)^{\prime}, i=1,2,3, k=1,2, \ldots, n$ em que $X_{i k j}, j=1,2$ denota a $j$-ésima medida associada à $k$-ésima unidade experimental sob o $i$-ésimo nível do fator $G$. Os vetores $\mathbf{X}_{i k}$ foram selecionadas segundo as distribuições dadas na Tabela 10.

Tabela 10: Distribuição das variáveis aleatórias $X_{i j k}$.

\begin{tabular}{|c|c|c|c|c|}
\hline \multirow[b]{2}{*}{$X_{12 k}$} & \multicolumn{3}{|c|}{$X_{11 k}$} & \multirow[b]{2}{*}{ Total } \\
\hline & 0 & 1 & 2 & \\
\hline 0 & $1 / 16$ & $1 / 16$ & 0 & $2 / 16$ \\
\hline 1 & $1 / 16$ & $1 / 16$ & $4 / 16$ & $6 / 16$ \\
\hline 2 & $6 / 16$ & $2 / 16$ & 0 & $8 / 16$ \\
\hline \multirow[t]{2}{*}{ Total } & $8 / 16$ & $4 / 16$ & $4 / 16$ & 1 \\
\hline & \multicolumn{3}{|c|}{$X_{21 k}$} & \\
\hline$X_{22 k}$ & 0 & 1 & 2 & Total \\
\hline 0 & 0 & 0 & $1 / 16$ & $1 / 16$ \\
\hline 1 & $2 / 16$ & $1 / 16$ & $3 / 16$ & $6 / 16$ \\
\hline 2 & $4 / 16$ & $3 / 16$ & $2 / 16$ & $9 / 16$ \\
\hline \multirow[t]{2}{*}{ Total } & $6 / 16$ & $4 / 16$ & $6 / 16$ & 1 \\
\hline & \multicolumn{3}{|c|}{$X_{31 k}$} & \\
\hline$X_{32 k}$ & 0 & 1 & 2 & Total \\
\hline 0 & $1 / 16$ & $1 / 16$ & 0 & $2 / 16$ \\
\hline 1 & $1 / 16$ & $1 / 16$ & $4 / 16$ & $6 / 16$ \\
\hline 2 & $6 / 16$ & $2 / 16$ & 0 & $8 / 16$ \\
\hline Total & $8 / 16$ & $4 / 16$ & $4 / 16$ & 1 \\
\hline
\end{tabular}

As funções de distribuição marginais normalizadas $\left(F_{i j}\right)$ para cada combinação dos níveis dos fatores $G$ e $R$ são dadas na Tabela 11 .

As hipóteses de inexistência de efeito principal dos fatores e de inexistência de efeito de interação entre os fatores são formuladas em termos destas funções de distribuição. Por exemplo, a hipótese de inexistência de interação é dada por

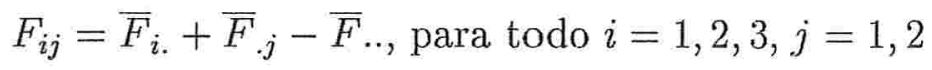


Tabela 11: Distribuições marginais normalizadas de $X_{i k j}$.

\begin{tabular}{lcccc}
\hline$X_{i j k}$ & & 0 & 1 & 2 \\
\cline { 1 - 1 } \cline { 5 - 5 }$F_{11}$ & & 0.250 & 0.625 & 0.875 \\
$F_{12}$ & & 0.063 & 0.313 & 0.750 \\
$F_{21}$ & & 0.188 & 0.500 & 0.813 \\
$F_{22}$ & & 0.031 & 0.250 & 0.719 \\
$F_{31}$ & & 0.250 & 0.625 & 0.875 \\
$F_{32}$ & & 0.063 & 0.313 & 0.750 \\
\hline
\end{tabular}

$\operatorname{com} \bar{F}_{i .}, \bar{F}_{. j}$ e $\bar{F}_{. .}$definidos como na Seção 3 . Sejam $F_{i j}^{0}=\bar{F}_{i .}+\bar{F}_{. j}-\bar{F}_{\text {... os va- }}$ lores esperados de $F_{i j}$ sob esta hipótese, calculados a partir das funções de distribuição normalizadas dadas na Tabela 10. Como exemplo, tome

$$
\begin{aligned}
F_{11}^{0}(0) & =\bar{F}_{1 .}(0)+\bar{F}_{.1}(0)-\bar{F} . .(0)=1 / 2(0.250+0.063) \\
& +1 / 3(0.250+0.188+0.250) \\
& -1 / 6(0.250+0.063+0.188+0.031+0.250+0.063)=0.245
\end{aligned}
$$

o valor esperado de $F_{i j l}$ sob a hipótese de inexistência de interação entre os fatores $G$ e $R$. Calculando essas quantidades para todas as funções de distribuição envolvidas e todos os possíveis valores de $X_{i j k}$ obtemos os valores da Tabela 12 .

Tabela 12: Valores esperados das distribuições marginais normalizadas de $X_{i k j}$

\begin{tabular}{ccccc}
\hline & & \multicolumn{3}{c}{$X_{i j k}$} \\
\cline { 3 - 5 } & & 0 & 1 & 2 \\
\cline { 1 - 1 }$F_{11}^{0}$ & & 0.245 & 0.615 & 0.870 \\
$F_{12}^{0}$ & & 0.068 & 0.323 & 0.755 \\
$F_{21}^{0}$ & & 0.198 & 0.521 & 0.823 \\
$F_{22}^{0}$ & & 0.021 & 0.229 & 0.708 \\
$F_{31}^{0}$ & & 0.245 & 0.615 & 0.870 \\
$F_{32}^{0}$ & & 0.068 & 0.323 & 0.755 \\
\hline
\end{tabular}

Comparando esses valores com os respectivos $F_{i j}$ da Tabela 11, nota-se que a hipótese (14) não é válida, porém a diferença entre as funções de distribuição correspondentes é muito pequena, de forma que a detecção desse efeito deve requerer tamanhos amostrais 
grandes. As hipóteses de inexistência de efeito principal dos fatores $G$ e $R$ também não são válidas, porém as diferenças entre as funções de distribuição normalizadas e seus valores esperados sob a hipótese nula são maiores, de forma que os efeitos deveriam ser detectados com maior facilidade. A Tabela 13 mostra os efeitos relativos de tratamento $p_{i j}$ calculados para cada combinação dos fatores envolvidos.

Tabela 13: Efeitos relativos de tratamento para as variáveis $X_{i j k}$.

\begin{tabular}{|c|c|c|c|}
\hline \multirow[b]{2}{*}{$R$} & \multicolumn{3}{|c|}{$G$} \\
\hline & 1 & 2 & 3 \\
\hline 1 & 0.398 & 0.412 & 0.398 \\
\hline 2 & 0.467 & 0.474 & 0.467 \\
\hline
\end{tabular}

Quando escores são atribuídos às classes de resposta e o procedimento paramétrico é utilizado, as hipóteses mencionadas são formuladas em termos das médias $\mu_{i j}=E\left(Y_{i k j}\right)$, com $Y_{i k j}$ denotando o escore atribuído à observação $X_{i k j}$. Nesta simulação utilizamos 3 sistemas de pontuação, ( $\left.\begin{array}{lll}0 & 1 & 2\end{array}\right),\left(\begin{array}{lll}0 & 1 & 5\end{array}\right)$ e ( $\left(\begin{array}{lll}0 & 4 & 5\end{array}\right)$. Para cada um apresentamos as médias $\mu_{i j}$, na Tabela 14.

Tabela 14: Médias de $Y_{i k j}$.

\begin{tabular}{llcccccc}
\cline { 1 - 6 } Escores & & $\mu_{11}$ & $\mu_{12}$ & $\mu_{21}$ & $\mu_{22}$ & $\mu_{31}$ & $\mu_{32}$ \\
\cline { 1 - 4 }$(0,1,2)$ & & 0.750 & 1.375 & 1.000 & 1.500 & 0.750 & 1.375 \\
$(0,1,5)$ & & 1.500 & 2.875 & 2.125 & 3.188 & 1.500 & 2.875 \\
$(0,4,5)$ & & 2.250 & 4.000 & 2.875 & 4.313 & 2.250 & 4.000 \\
\hline
\end{tabular}

Para os 3 sistemas de pontuação, a hipótese de inexistência de interação entre os fatores,

$$
\mu_{i j}=\mu_{i .}+\mu_{. j}-\mu_{. .}, \text {para todo } i=1,2,3, j=1,2
$$

não é válida. O mesmo ocorre para as hipóteses de inexistência de efeito dos fatores $G$ e $R$.

Para analisar o poder associado a cada um destes testes, calculamos a taxa de rejeição de $H_{0}$, com níveis $\alpha=1 \%$ e $5 \%$, utilizando 1000 amostras geradas sob o planejamento 
descrito anteriormente, para $\mathrm{n}=10,20,50,100,500$. Os resultados são mostrados nas Tabelas 15 a 20.

Tabela 15: Taxa de rejeição da hipótese nula de inexistência de efeito do fator $G(\alpha=1 \%)$.

\begin{tabular}{|c|c|c|c|c|c|}
\hline \multirow[b]{2}{*}{$\mathrm{n}$} & \multicolumn{3}{|c|}{$\begin{array}{l}\text { Testes paramétricos } \\
\text { com escores }\end{array}$} & \multicolumn{2}{|c|}{$\begin{array}{l}\text { Testes não- } \\
\text { paramétricos }\end{array}$} \\
\hline & $\left(\begin{array}{lll}0 & 1 & 2\end{array}\right)$ & $\left(\begin{array}{lll}0 & 1 & 5\end{array}\right)$ & $\left(\begin{array}{lll}0 & 4 & 5\end{array}\right)$ & Wald & ANOVA \\
\hline 10 & 0.032 & 0.016 & 0.017 & 0.075 & 0.053 \\
\hline 20 & 0.078 & 0.078 & 0.039 & 0.109 & 0.101 \\
\hline 50 & 0.373 & 0.307 & 0.206 & 0.359 & 0.388 \\
\hline 100 & 0.749 & 0.688 & 0.535 & 0.750 & 0.767 \\
\hline 500 & 1.000 & 1.000 & 1.000 & 1.000 & 1.000 \\
\hline
\end{tabular}

Tabela 16: Taxa de rejeição da hipótese nula de inexistência de efeito do fator $R$ ( $\alpha=1 \%$ ).

\begin{tabular}{|c|c|c|c|c|c|}
\hline & \multicolumn{3}{|c|}{$\begin{array}{c}\text { Testes paramétricos } \\
\text { com escores }\end{array}$} & \multicolumn{2}{|c|}{$\begin{array}{l}\text { Testes não- } \\
\text { paramétricos }\end{array}$} \\
\hline $\mathrm{n}$ & $\left(\begin{array}{lll}0 & 1 & 2\end{array}\right)$ & $\left(\begin{array}{lll}0 & 1 & 5\end{array}\right)$ & $(045)$ & Wald & ANOVA \\
\hline 10 & 0.398 & 0.082 & 0.314 & 0.433 & 0.433 \\
\hline 20 & 0.810 & 0.289 & 0.832 & 0.791 & 0.791 \\
\hline 50 & 1.000 & 0.840 & 1.000 & 1.000 & 1.000 \\
\hline 100 & 1.000 & 0.996 & 1.000 & 1.000 & 1.000 \\
\hline 500 & 1.000 & 1.000 & 1.000 & 1.000 & 1.000 \\
\hline
\end{tabular}

Tabela 17: Taxa de rejeição da hipótese nula de inexistência de interação ( $\alpha=1 \%)$.

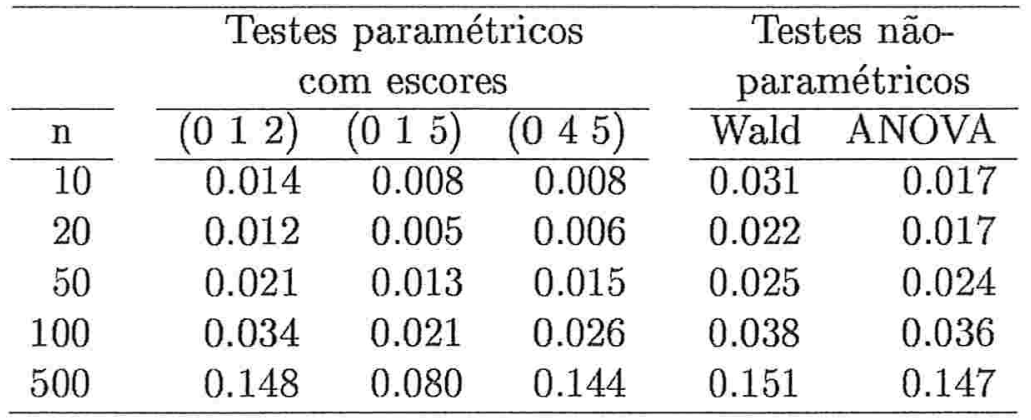

De acordo com os resultados, as taxas de rejeição de $H_{0}$ com a estatística tipo-ANOVA são similares às obtidas com a utilização do escore $\left(\begin{array}{lll}0 & 1 & 2\end{array}\right)$ sob o modelo paramétrico. 
Tabela 18: Taxa de rejeição da hipótese nula de inexistência de efeito do fator $G(\alpha=5 \%)$.

\begin{tabular}{|c|c|c|c|c|c|}
\hline \multirow[b]{2}{*}{$\mathrm{n}$} & \multicolumn{3}{|c|}{$\begin{array}{c}\text { Testes paramétricos } \\
\text { com escores }\end{array}$} & \multicolumn{2}{|c|}{$\begin{array}{l}\text { Testes não- } \\
\text { paramétricos }\end{array}$} \\
\hline & $\left(\begin{array}{lll}0 & 1 & 2\end{array}\right)$ & $\left(\begin{array}{lll}0 & 1 & 5\end{array}\right)$ & $\left(\begin{array}{lll}0 & 4 & 5\end{array}\right)$ & Wald & ANOVA \\
\hline 10 & 0.132 & 0.138 & 0.098 & 0.170 & 0.163 \\
\hline 20 & 0.260 & 0.250 & 0.195 & 0.272 & 0.279 \\
\hline 50 & 0.612 & 0.615 & 0.516 & 0.698 & 0.627 \\
\hline 100 & 0.913 & 0.891 & 0.830 & 0.903 & 0.913 \\
\hline 500 & 1.000 & 1.000 & 1.000 & 1.000 & 1.000 \\
\hline
\end{tabular}

Tabela 19: Taxa de rejeição da hipótese nula de inexistência de efeito do fator $R(\alpha=5 \%)$.

\begin{tabular}{|c|c|c|c|c|c|}
\hline & \multicolumn{3}{|c|}{$\begin{array}{c}\text { Testes paramétricos } \\
\text { com escores }\end{array}$} & \multicolumn{2}{|c|}{$\begin{array}{l}\text { Testes não- } \\
\text { paramétricos }\end{array}$} \\
\hline $\mathrm{n}$ & $\left(\begin{array}{lll}0 & 1 & 2\end{array}\right)$ & $\left(\begin{array}{lll}0 & 1 & 5\end{array}\right)$ & $\left(\begin{array}{lll}0 & 4 & 5\end{array}\right)$ & Wald & ANOVA \\
\hline 10 & 0.670 & 0.260 & 0.692 & 0.657 & 0.657 \\
\hline 20 & 0.935 & 0.591 & 0.966 & 0.917 & 0.917 \\
\hline 50 & 1.000 & 0.961 & 1.000 & 1.000 & 1.000 \\
\hline 100 & 1.000 & 0.999 & 1.000 & 1.000 & 1.000 \\
\hline 500 & 1.000 & 1.000 & 1.000 & 1.000 & 1.000 \\
\hline
\end{tabular}

Tabela 20: Taxa de rejeição da hipótese nula de inexistência de interação ( $\alpha=5 \%)$.

\begin{tabular}{|c|c|c|c|c|c|}
\hline \multirow[b]{2}{*}{$\mathrm{n}$} & \multicolumn{3}{|c|}{$\begin{array}{c}\text { Testes paramétricos } \\
\text { com escores }\end{array}$} & \multicolumn{2}{|c|}{$\begin{array}{l}\text { Testes não- } \\
\text { paramétricos }\end{array}$} \\
\hline & $\left(\begin{array}{lll}0 & 1 & 2\end{array}\right)$ & $\left(\begin{array}{lll}0 & 1 & 5\end{array}\right)$ & $\left(\begin{array}{lll}0 & 4 & 5\end{array}\right)$ & Wald & ANOVA \\
\hline 10 & 0.047 & 0.044 & 0.053 & 0.078 & 0.062 \\
\hline 20 & 0.049 & 0.049 & 0.066 & 0.063 & 0.055 \\
\hline 50 & 0.083 & 0.089 & 0.078 & 0.091 & 0.090 \\
\hline 100 & 0.119 & 0.104 & 0.138 & 0.120 & 0.118 \\
\hline 500 & 0.332 & 0.281 & 0.407 & 0.331 & 0.324 \\
\hline
\end{tabular}

A estatística de Wald, por ser mais liberal, apresenta níveis maiores de rejeição que a estatística tipo-ANOVA.

Com os diferentes esquemas de pontuação, não somente as médias mas também as variâncias e as covariâncias são alteradas, o que obviamente influencia os resultados. Como exemplo, note as diferenças entre as taxas de rejeição da hipótese de inexistência 
de efeito de fator $R$ sob os escores $\left(\begin{array}{lll}0 & 1 & 2\end{array}\right)$ e $\left(\begin{array}{lll}0 & 1 & 5\end{array}\right)$, para $\mathrm{n}=10$ e $\mathrm{n}=20$, nas Tabelas 16 e 19. Estes resultados indicam que a escolha do sistema de escore para as categorias de resposta pode resultar em uma perda significativa de poder do teste paramétrico, o que favorece os testes não-paramétricos.

Uma vez que a teoria desenvolvida permite a análise de planejamentos não-balanceados ou com dados omissos (missing data), em pesquisas futuras, a influência da falta de algumas observações nos resultados dos testes do modelo não-paramétricos pode ser analisada. Outro tópico interessante, como complemento da análise descritiva fornecida pela comparação dos efeitos relativos de tratamento, seria a estimação de alguma medida da variabilidade desses efeitos. Brunner and Puri (2001), em um trabalho recente, sugerem a construção de intervalos de confiança para os efeitos não-paramétricos de tratamento utilizando estimadores consistentes dessas variâncias também baseados em postos das observações originais.

\section{Aspectos Computacionais}

Para o ajuste do modelo não-paramétrico, Brunner and Langer (2000) disponibilizaram subrotinas SAS que consideram modelos com até 2 fatores intraindivíduos e 2 fatores entreindivíduos (http:/www.ams.med.uni-goettingen.de/Projekte/LD/MakrosLD.html). Como resultados, apresentam as estimativas dos efeitos de tratamento e o cálculo das estatísticas de teste apresentadas na Seção 4 para investigar as hipóteses usuais de inexistência de interação e inexistência dos efeitos principais dos fatores envolvidos. Essas subrotinas não permitem a especificação de outras hipóteses .

Com o objetivo de testar hipóteses do tipo $\mathbf{C p}=\mathbf{0}$ em geral, i.e., com matrizes $\mathrm{C}$ diferentes daquelas definidas na Seção 2, construímos subrotinas em Visual Basic para Excel (Microsoft Excel 2000) que permitem o ajuste de modelos em dois casos: experimentos desenhados somente com fatores intra indivíduos (ou de medidas repetidas) e experimentos que também incluam fatores entre indivíduos (ou de estratificação).

Essas subrotinas fornecem estimativas dos efeitos relativos de tratamento definidos na Seção 3, para cada combinação dos níveis dos fatores envolvidos. Apresentam também as estatísticas de teste de Wald e tipo-ANOVA para as hipóteses usuais. Para experimentos 
sem fatores de estratificação, a hipótese automaticamente testada é a de inexistência de efeito do fator de medidas repetidas, ou seja, a hipótese $F_{i}=\bar{F}$. $i=1, \ldots, t$, com $t$ denotando o número de níveis do fator de repetição.

O programa possibilita ainda o cálculo de estatísticas de teste para outras hipóteses do tipo $\mathbf{C F}=0, \mathbf{F}=\left(F_{1}, F_{2}, \ldots, F_{t}\right)^{\prime}$. A matriz $\mathbf{C}$ de contrastes correspondente com dimensão $r \times t, 1 \leq r \leq t-1$ deve ser especificada pelo usuário. Além disso, as linhas da matriz $\mathbf{C}$ devem ser linearmente independentes. A ordem dos coeficientes associados às respectivas funções distribuição $F_{i}, i=1,2, \ldots, t$ deve seguir a ordem estabelecida na apresentação do conjunto de dados.

Considere como exemplo, o conjunto da Tabela 21. Com essa disposição das colunas dos dados na planilha, o vetor $\mathbf{F}$ das funções distribuição será definido por $\mathbf{F}=$ $\left(F_{1}, F_{2}, F_{3}\right)^{\prime}$. Para testar a hipótese de igualdade das funções $F_{1}$ e $F_{2}$, ou seja, para testar a hipótese $F_{1}=F_{2}$, a matriz de contrastes correspondente é dada por $\mathbf{C}=\left[\begin{array}{lll}1 & -1 & 0\end{array}\right]$.

Tabela 21: Exemplo de disposição dos dados de entrada na planilha de ajuste do modelo não-paramétrico.

\begin{tabular}{|c|c|c|}
\hline Medida1 & Medida2 & Medida3 \\
\hline 1 & 2 & 4 \\
\hline 4 & 3 & 1 \\
\hline 1 & 2 & 3 \\
\hline . & . & . \\
\hline . & . & $\cdot$ \\
\hline . & . & . \\
\hline 1 & 1 & 2 \\
\hline
\end{tabular}

Para experimentos que possuam, adicionalmente, um fator de estratificação a saída usual apresenta estatísticas para testar as hipóteses de inexistência de efeitos principais dos fatores e de inexistência de efeito de interação entre eles. A matriz de contrastes para testar hipóteses do tipo $\mathbf{C F}=0$ deve ter dimensão $r \mathrm{x}(g . t), 1 \leq r \leq(g . t)-1$, com $t$ denotando o número de níveis do fator de repetição e $g$ o número de níveis do fator de estratificação. Como no caso anterior, as linhas de $\mathbf{C}$ devem ser linearmente independentes.

A ordem das respectivas funções distribuição $F_{i j}, i=1,2, \ldots, g, j=1,2, \ldots, t$ é a 
ordem lexicográfica, ou seja,

$$
\mathbf{F}=\left(\mathbf{F}_{\mathbf{1}}, \mathbf{F}_{\mathbf{2}}, \ldots, \mathbf{F}_{\mathbf{g}}\right), F_{i .}=\left(F_{i 1}, F_{i 2}, \ldots, F_{i t}\right)
$$

com a ordem dos níveis dos fatores entre indivíduos e dos fatores intra indivíduos definida conforme a apresentação do conjunto de dados na planilha.

Como exemplo, considere o conjunto de dados da Tabela 22.

Tabela 22: Exemplo da disposição do conjunto de dados de entrada na planilha de ajuste do modelo não-paramétrico.

\begin{tabular}{|c|c|c|c|}
\hline Estrato & Medida1 & Medida2 & Medida3 \\
\hline $\mathrm{B}$ & 1 & 2 & 1 \\
\hline A & 3 & 2 & 1 \\
\hline $\mathrm{C}$ & 1 & 1 & 2 \\
\hline $\mathrm{C}$ & 2 & 1 & 1 \\
\hline $\mathrm{A}$ & 3 & 2 & 3 \\
\hline $\mathrm{C}$ & 1 & 1 & 1 \\
\hline B & 2 & 1 & 3 \\
\hline B & 1 & 2 & 1 \\
\hline $\mathrm{A}$ & 2 & 2 & 3 \\
\hline
\end{tabular}

A ordem correspondente dos níveis do fator entre indivíduos (Estrato), é dada por $\mathrm{i}=1 \Rightarrow \mathrm{B}, \mathrm{i}=2 \Rightarrow \mathrm{A}, \mathrm{i}=3 \Rightarrow \mathrm{C}$. Para o fator intra indivíduos, $\mathrm{j}=1 \Rightarrow$ Medida $1, \mathrm{j}=2 \Rightarrow$ Medida2, $\mathrm{j}=3 \Rightarrow$ Medida3. Assim, $\mathbf{F}$ é dada por $\mathbf{F}=\left(F_{11}, F_{12}, F_{13}, \ldots, F_{31}, F_{32}, F_{33}\right) \prime$. A construção da matriz de contrastes para hipóteses especificados pelo usuário deve considerar essa ordem na atribuição dos respectivos coeficientes associados às funções distribuição $F_{i j}$.

Como exemplo, a hipótese de igualdade das funções distribuição $F_{1 j}, j=1,2,3$, ou seja, inexistência de diferença na distribuição da resposta conforme o nível do fator de repetição no estrato $B$, é dada por $C F=0$ com

$$
\mathbf{C}=\left[\begin{array}{ccccccccc}
1 & -1 & 0 & 0 & 0 & 0 & 0 & 0 & 0 \\
0 & 1 & -1 & 0 & 0 & 0 & 0 & 0 & 0
\end{array}\right]
$$

As estatísticas para esses testes de hipóteses adicionais são apresentados na planilha de resultados com o título "User Specified Contrast". Esse procedimento é bastante útil 
em análises complementares como comparações múltiplas e testes de contrastes. Além disso, as hipóteses usuais de inexistência de efeitos principais de fator e de inexistência de efeito de interação entre eles, para experimentos com maior número de fatores, podem ser testadas construindo-se as matrizes $\mathbf{C}$ apropriadamente.

As planilhas com as macros implementadas podem ser obtidas em http://ime.usp.br/ prosa ou http://ime.usp.br/ jmsinger. 


\section{Apêndice}

Tabela A.1: Escores de Microinfiltração - Região Central.

\begin{tabular}{|c|c|c|c|c|c|c|c|}
\hline \multicolumn{4}{|c|}{ Campo Seco } & \multicolumn{4}{|c|}{ Campo Úmido } \\
\hline \multicolumn{4}{|c|}{ Examinador } & \multicolumn{4}{|c|}{ Examinador } \\
\hline Material & $\mathrm{A}$ & $\mathrm{B}$ & $\mathrm{C}$ & Material & $\mathrm{A}$ & $\mathrm{B}$ & $\mathrm{C}$ \\
\hline \multirow{12}{*}{ Allbond } & 0 & 2 & 0 & \multirow{12}{*}{ Allbond } & 2 & 2 & 2 \\
\hline & 2 & 2 & 1 & & 2 & 2 & 2 \\
\hline & 0 & 2 & 1 & & 0 & 2 & 1 \\
\hline & 2 & 2 & 2 & & 4 & 4 & 2 \\
\hline & 1 & 0 & 3 & & 1 & 1 & 1 \\
\hline & 2 & 1 & 2 & & 1 & 2 & 2 \\
\hline & 1 & 2 & 0 & & 2 & 2 & 1 \\
\hline & 2 & 2 & 1 & & 3 & 3 & 2 \\
\hline & 3 & 3 & 3 & & 1 & 0 & 1 \\
\hline & 0 & 3 & 0 & & 3 & 3 & 2 \\
\hline & 3 & 1 & 1 & & 3 & 3 & 1 \\
\hline & 1 & 2 & 2 & & 2 & 2 & 2 \\
\hline \multirow{12}{*}{ Optibond } & 0 & 2 & 0 & \multirow{12}{*}{ Optibond } & 0 & 0 & 0 \\
\hline & 1 & 1 & 1 & & 0 & 0 & 0 \\
\hline & 1 & 3 & 1 & & 0 & 0 & 1 \\
\hline & 3 & 3 & 4 & & 0 & 1 & 1 \\
\hline & 0 & 0 & 1 & & 1 & 2 & 1 \\
\hline & 2 & 2 & 1 & & 0 & 2 & 1 \\
\hline & 0 & 1 & 1 & & 1 & 2 & 2 \\
\hline & 1 & 1 & 1 & & 0 & 0 & 0 \\
\hline & 1 & 1 & 1 & & 0 & 0 & 1 \\
\hline & 0 & 2 & 0 & & 0 & 0 & 1 \\
\hline & 1 & 1 & 1 & & 1 & 0 & 1 \\
\hline & 1 & 1 & 1 & & 0 & 1 & 1 \\
\hline \multirow{12}{*}{ Scotchbond } & 0 & 0 & 3 & \multirow{12}{*}{ Scotchbond } & 3 & 4 & 3 \\
\hline & 1 & 2 & 1 & & 2 & 2 & 1 \\
\hline & 3 & 3 & 2 & & 3 & 4 & 3 \\
\hline & 3 & 3 & 2 & & 2 & 0 & 1 \\
\hline & 0 & 0 & 0 & & 3 & 3 & 3 \\
\hline & 2 & 3 & 1 & & 2 & 4 & 2 \\
\hline & 1 & 2 & 2 & & 3 & 3 & 0 \\
\hline & 2 & 2 & 3 & & 1 & 3 & 1 \\
\hline & 1 & 1 & 1 & & 3 & 4 & 4 \\
\hline & 0 & 0 & 1 & & 4 & 3 & 1 \\
\hline & 3 & 2 & 1 & & 3 & 2 & 3 \\
\hline & 1 & 1 & 1 & & 3 & 3 & 3 \\
\hline
\end{tabular}




\section{Referências Bibliográficas}

Akritas, M.G. and Arnold, S.F. (1994). Fully nonparametric hypotheses for factorial design I: Multivariate repeated measures designs. Journal of the American Statistical Association, 89, 336-343.

Akritas, M.G. and Brunner, E. (1997). A unified approach to rank tests for mixed models. Journal of Statistical Planning and Inference, 61, 249-277.

Brunner, E. and Langer, F. (2000). Nonparametric analysis of ordered categorical data in designs with longitudinal observations and small sample sizes. Biometrical Journal, 42, 663-675.

Brunner, E., Munzel, U. and Puri, M.L. (1999). Rank-score tests in factorial designs with repeated measures. Journal of Multivariate Analysis, 70, 286-317.

Brunner, E., Puri, M. L. (2001). Point and interval estimators for nonparametric treatment effects in designs with repeated measures. (http://www.ams.med. uni-goettingen.de/brunner/papers/cirmf1.pdf)

Crowder, M.J. and Hand, D.J. (1990). Analysis of repeated measures. New York: Chapman and Hall.

Diggle, P.J., Liang, K.Y. and Zeger, S.L. (1994). Analysis of longitudinal data. New York: Oxford University Press.

Ivanova, A. and Berger, V.W. (2001). Drawbacks to integer scoring for ordered categorical data. Biometrics, 57, 567-570.

Kruskal, W.H. and Wallis, W.A. (1952). The use of ranks on one-criterion variance analysis. Journal of the American Statistical Association, 47, 583-621.

Mann, H.B. and Whitney, D.R. (1947). On a test of whether one of two random variables is stocastically larger than the other. The Annals of Mathematical Statistics, 18, 50-60. 
Munzel, U. and Brunner, E. (2000). Nonparametric tests in the unbalanced multivariate one-way design. Biometrical Journal, 42, 837-854.

Singer, J.M. and Andrade, D.F. (2000). Analysis of Longitudinal Data. In Handbook of Statistics, volume 18: Bio-environmental and Public Health Statistics, eds. P.K. Sen and C.R. Rao. Amsterdam: North Holland, 115-160.

Witzel, M.F., Grande, R.H.M. and Singer, J.M. (2000). Bonding systems used for sealing: evaluation of microleakage. Journal of Clinical Dentistry, 11, 45-52. 